\title{
Relation between Hypoxic Markers P65, P50, CAIX, and Tumor Stages in Invasive Ductal Carcinoma Subtypes
}

\author{
Eman El-Abd ${ }^{1}$, Cecil A. Matta ${ }^{2}$, Manal Sheta ${ }^{3}$,Yasser El-Kerm ${ }^{4}$, Mohammed Samy \\ Afifi $^{5}$, Traki Benhassine ${ }^{6}$, Sarah Meftahi ${ }^{7}$, Shimaa Sakr ${ }^{8}$ and Baseem Elsherbini ${ }^{9}$ \\ ${ }^{1,8}$ Molecular Biology Department, Medical Technology Centre (MTC), Medical Research Institute \\ (MRI), Alexandria University, Egypt \\ ${ }^{2}$ Zoology Department, Faculty of science, Alexandria University, Egypt \\ ${ }^{3}$ Pathology Department, MRI, Alexandria University, Egypt \\ ${ }^{4}$ Cancer research and management department, MRI, Alexandria University, Egypt \\ ${ }^{5,9}$ Immunology Department, MRI, Alexandria University, Egypt \\ ${ }^{6}$ Laboratory of Cellular and Molecular Biology, Faculty of Biological Sciences, USTHB, Algiers, Algeria \\ ${ }^{7}$ Laboratoire de Biologie Cellulaire et Moléculaire, Faculté Des Sciences Biologiques (FSB), Université \\ des Sciences et de La Technologie Houari Boumedienne (USTHB), Algeria
}

Correspondence should be addressed to: Eman El-Abd; elabdeman@yahoo.com

Received Date: 31 January 2014; Accepted Date: 10 July 2014; Published Date: 25 February 2015

Academic Editor: Ahmed F. Salem

Copyright (C) 2015. Eman El-Abd, Cecil A. Matta, Manal Sheta ,Yasser El-Kerm, Mohammed Samy Afifi, Traki Benhassine, Sarah Meftahi, Shimaa Sakr and Baseem Elsherbini . Distributed under Creative Commons CC-BY 4.0

\begin{abstract}
Background: Nuclear factor-kappa B (NF-?B) family comprises 5 members (p50, p52, relA/p65, c-rel and relB) which are induced in response to a wide variety of stimuli including hypoxia. Continuous activation of NF-? $\mathrm{B}$ is an important factor in the onset and progression of breast carcinoma. Hypoxia also induces carbonic anhydrase 9 (CAIX) that regulates $\mathrm{pH}$ and is linked to poor prognosis in breast cancer. Research motivation: The current study aims to investigate the relation between hypoxic markers, p65, p50, CAIX and tumor stage in IDC (invasive ductal carcinoma) subtypes. Research design/methodology: The study included 31 IDC patients. Breast tissues collected during surgery and classified according to estrogen receptor alpha (ER? ?, progesterone receptor (PR), and human epidermal growth factor receptor-2 (HER-2) status. Normal breast tissues were also collected to serve as self controls. Nuclear protein extracted and both RelA/p65 and p50 protein assessed by ready to use enzyme-linked immunosorbent assay (ELISA) and a transcription factor assay kits; respectively. CAIX protein expression was detected by blotting techniques. Main findings: RelA/p65 concentration significantly increased in breast carcinoma ( $\mathrm{p}=0.028$ ) irrelevant to tumor stage, size, grade, nodal status, $\mathrm{p} 50$, CAIX or IDC subtypes. $\mathrm{P} 50$ binding activity significantly increased with higher tumor grade $(\mathrm{P}=$ $0.042)$. A significant inverse correlation was observed between $\mathrm{p} 50$ and $\mathrm{ER}$ ? $(\mathrm{r}=-0.53, \mathrm{p}=$ $0.002)$ and between CAIX and the number of the involved lymph nodes $(r=-0.42, p=0.020)$. Implications: Although no relation was observed between p65, p50, and CAIX, binding activity of p50 and CAIX concentration might be used as prognostic markers in IDC.
\end{abstract}

Keywords: Nuclear factor-kappa B (NF-? B), invasive ductal carcinoma (IDC) subtypes, carbonic anhydrase 9 (CAIX)

Cite this Article as: Eman El-Abd, Cecil A. Matta, Manal Sheta ,Yasser El-Kerm, Mohammed Samy Afifi, Traki Benhassine, Sarah Meftahi, Shimaa Sakr and Baseem Elsherbini (2015)," Relation between Hypoxic Markers P65, P50, CAIX, and Tumor Stages in Invasive Ductal Carcinoma Subtypes ", Advances in Cancer Research \& Treatment, Vol. 2015 (2015), Article ID 965854, DOI: 10.5171/2015.965854 


\section{Introduction}

Hypoxia and acidosis are hallmark features of aggressive breast cancer phenotypes (Knowles \& Harris, 2001). Up to $1.5 \%$ of the human genome is responsive to hypoxia at the transcriptional level. The hypoxia gene expression profile varies according to the tumor type. Thus, several studies were conducted to identify the gene signatures that have prognostic significance in breast cancer (Favaro et al., 2011). Hypoxia inducible factor-1 (HIF-1); the main sensor of hypoxia; regulates key aspects in cancer biology including $\mathrm{pH}$ regulation through binding to a hypoxia responsive element in the carbonic anhydrase 9 (CAIX) gene (Thiry et al., 2006). Hypoxia also differentially regulates the activity of NF-kB (nuclear factor-kappa B) pathways and subsequent gene expression (Taylor \& Cummins, 2009; Culver et al., 2010). Both NF-? ? B and CAIX negatively influence prognosis of patients with breast cancer (Wycoff et al., 2000; Chia et al., 2001; Span et al., 2003; Montagut et al., 2006; Trastour et al., 2007, Jana et al., 2012; Shapochkka et al., 2012).

The transcription factor NF-?B family consists of five subunits: NF-?B1 (p50/p105), NF-kB2 (p52/p100), RelA (p65), RelB, and c-Rel (Ahn \& Aggarwal, 2005). The five subunits differ in the structure of the C-terminus region. NF-kB proteins have a structurally conserved $\mathrm{N}$ terminal 300-amino acid region called rel homology region (RHR), which contains the dimerisation, nuclear localization, and DNA binding domains. The RHR is responsible for dimerisation, nuclear translocation (NL), DNA binding and regulation of NF-? B through interaction with its inhibitor, I? B (inhibitory kappa B kinases). The c-Rel, Rel $\mathrm{B}$, and Rel A proteins also have a C-terminal non homologous transactivation domain (TAD) that strongly activates transcription from NF-?B binding sites. P50 homodimers lack the transcription activation domain but still bind to ? B-consensus sites and therefore function as transcription repressors (May \& Ghosh, 1997). NF-?B have been shown to be expressed ubiquitously in the cytoplasm of all cell types in the form of homo- or heterodimers bound to specific inhibitory molecules; IkB. Under stimulation, I? $\mathrm{B}$ is degraded and NF-? $\mathrm{B}$ is activated and translocated to the nucleus (Koong et al., 1994; Ahn \& Aggarwal, 2005; Pereira \& Oakley, 2008). The activation of NF-?B leads to the stimulation of more than 500 genes involved in many physiological responses including cellular proliferation, differentiation, survival, inflammation, and transformation (Gupta et al., 2010).

NF-?B family appears to play an early and critical role in the malignant transformation of mammary glands and was implicated in mediating the development and progression of breast cancer (Wu \& Kral, 2005; Montagut et al., 2006). NF-? B activation was also reported as an important determinant for clinical behavior and was postulated as therapeutic target for human epidermal growth factor receptor 2/proto-oncogene neu (Her$2 /$ neu) positive and estrogen receptor(ER) negative breast cancer subtype (Biswas et al., 2001; Zhou et al., 2005a; Singh et al., 2007). Many studies focused on survival and proliferation pathways involving NF-? B and ER crosstalk (Biswas et al., 2004; Basak \& Hoffmann, 2008). Rel A (p65 subunit) is not only induced by hypoxia but also can modulate the expression and activity of hypoxia inducible factor-1 (HIF-1) which is the main sensor of hypoxia (Déry et al., 2005; Kenneth \& Rocha, 2008). Rel A is also the only subunit of NF-? B family that controls the metabolism and energy production in tumor cell (Johnson \& Perkins, 2012). P50 overexpression and increased p65 phosphorylation were linked to antiestrogen resistance (Zhou et al., 2005b; Yde et al., 2012). Moreover, p50 indicates early relapse in ER-positive breast cancer tumors (Zhou et al., 2005a \&b).

CAIX is an enzymatic transmembrane protein which plays a role in the acidification of the extracellular environment (Nogradi, 1998). CAIX protein is normally expressed in the alimentary tract and its associates, but is ectopically induced and regulated by HIF-1 in many 
types of tumors including breast cancer (Thiry et al., 2006). It potentiates tumor growth, survival under hypoxic conditions, extracellular matrix (ECM) breakdown and cellular invasion (Parks et al., 2011). The highest expression of CAIX was detected in triple negative breast cancer (TNBC) especially basal-like (9 times higher) tumors. CAIX expression correlated with bad prognostic factors such as higher grade, larger size, hormone receptors negativity, Her-2 positivity, tumor necrosis, higher relapse rate, and worse overall survival (OS) (Chia et al., 2001; Wykoff et al., 2001; Bartosova et al., 2002; Hussain et al., 2007; Tan et al., 2009). CAIX is also constitutively expressed in advanced breast cancer and metastasis by hypoxiaindependent mechanisms (Robey et al., 2005; Tafreshi et al., 2012).

Far to our knowledge, no study investigated the relation between the hypoxic markers p65, p50, CAIX, and tumor stags in invasive ductal carcinoma (IDC) subtypes. Therefore, this study was designed to investigate this possible relationship and their impacts on patients' outcomes.

\section{Research Design and Methodology}

The study included 31 patients (sample size was based on previously published work) with IDC randomly selected from cases presented to the Cancer Management and Research Department of Medical Research Institute (MRI), Alexandria, Egypt (from February 2007 to August 2009). Clinical diagnosis, treatment, and clinical follow up were performed according to ElAbd et al. (2012). Prognostic index was calculated using the Nottingham prognostic index (NPI) (Haybittle et al., 1982). All subjects recruited according to the ethical rules approved by the ethical committee of the MRI based on Belmont report (http://www.hhs.gov/ohrp/

humansubjects/guidance/belmont.htm).

Preoperative tumor evaluation was performed using fine needle aspiration cytology (FNAC) and/or excision biopsy. All mastectomy specimens were subjected to routine histopathological examination to determine tumor type (WHO, 2003), grade (Bloom and Richardson, 1957), stage (according to the American Joint Committee of Cancer (AJCC) (Greene et al., 2002), and hormonal receptor status (estrogen receptor: ER; progesterone receptor: PR), and Her-2/neu status (ElAbd et al., 2014). Tumor and normal tissue specimens (far from tumor site to serve as self-controls) were collected during the elective surgery by the pathologist and stored at $-80^{\circ} \mathrm{C}$ until use.

\section{Protein Extraction and SDS-PAGE}

Breast tissue samples (0.2 gram) were gently homogenized in $1 \mathrm{X}$ hypotonic buffer (20 mM Tris- $\mathrm{HCl}, \mathrm{pH} 7.4,10 \mathrm{mM} \mathrm{NaCl}$, and $3 \mathrm{mM} \mathrm{MgCl} 2$ ) and incubated for 15 minutes on ice. Ten percent NP40 $(25 \mu \mathrm{L})$ (SigmaAldrich, Switzerland) was added and mixed by vortex for 10 seconds at maximum speed then the homogenate was centrifuged $(3000 \mathrm{rpm})$ for 10 minutes at $4^{\circ} \mathrm{C}$. The supernatant (containing the cytoplasmic fraction) was transferred into a clean micro-centrifuge tube (used for CAIX detection).

The pellet was re-suspended in Cell Extraction Buffer (CEB) [CEB; $10 \mathrm{mM}$ Tris (pH 7.4), $100 \mathrm{mM} \mathrm{NaCl}, 1 \mathrm{mM}$ EDTA, $1 \mathrm{mM}$ EGTA, $1 \mathrm{mM} \mathrm{NaF}, 20 \mathrm{mM} \mathrm{Na} 4 \mathrm{P}_{2} \mathrm{O}_{7}, 2 \mathrm{mM}$ $\mathrm{Na}_{3} \mathrm{VO}_{4}, 1 \%$ Triton $\mathrm{X}-100,10 \%$ glycerol, $0.1 \% \quad$ SDS, $\quad 0.5 \%$ deoxycholate supplemented with $1 \mathrm{mM}$ PMSF (stock is $0.3 \mathrm{M}$ in DMSO)] (BioSource International, Inc., USA) containing protease inhibitors $(250 \mu \mathrm{L}$ of protease inhibitor cocktail per $5 \mathrm{~mL}$ CEB) (Sigma-Aldrich, Germany) for 30 minutes on ice. Subsequently, the mixture centrifuged for 30 minutes at maximum speed at $4^{\circ} \mathrm{C}$. The supernatant (nuclear fraction) transferred into a clean microcentrifuge tube (used for immunological assays to detect $\mathrm{NF}-\mathrm{kB} / \mathrm{p} 65$ and $\mathrm{p} 50$ subunits).

Protein concentration (for cytoplasmic and nuclear fractions) was determined using Biuret reagent kit (Biodiagnostic Co Ltd; Egypt) according to the manufacturer instructions. Aliquots were kept at $-80^{\circ} \mathrm{C}$ 
until used. The cytoplasmic protein extracts were fractionated on 12\% SDSPAGE (Sambrook et al., 1989) to verify its integrity and to trans-blot in further western and dot blot analyses

\section{Cell Culture}

The cervical carcinoma cell line HeLa cells (VACSERA, Egypt) were cultured in complete medium containing DMEM (Dulbecco's Modified Eagle Medium; Lonza, Belguim) supplemented with $10 \%$ fetal calf serum (Gibco, UK), 1\% antibiotics (streptomycin, penicillin; Gibco, UK), and 1\% L-Glutamine (Biowhiyyaker Europe, Belgium). Cells were used as positive control for CAIX after triggering its expression by high cell density and hypoxia imitation by $\mathrm{CoCl}_{2}$. Cells were grown for full confluence in complete medium containing $240 \mathrm{mM} \mathrm{CoCl}_{2}$ (Sigma, USA) (as hypoxia-mimetic agent that artificially induce hypoxia and activate the cell density-dependent CAIX expression via separate but interdependent pathway of PI3K (phosphoinositide 3-kinase) activation and a minimal level of HIF-1?; Kaluz et al., 2002) at $37{ }^{\circ} \mathrm{C}$ and $5 \% \mathrm{CO}_{2}$ for 24 hours. The medium was then removed and cells were washed twice using $1 \mathrm{X}$ PBS (Gibco, UK). Cells were Trypsinized (Freshney, 2010) and the pellet was subjected to repeated cycles (3 cycles) of freezing (at -80C) and thawing (on ice). Subsequently, the pellet was suspended in $300 \mathrm{ml}$ of CEB and kept on ice for 30 minutes with vortex at 10 minutes intervals. Following incubation, the suspension was centrifuged at 13,000 rpm for 10 minutes and the supernatant (containing the whole protein extract) was collected and protein concentration was detected then kept at $-80 \mathrm{C}$ till used to assay CAIX.

\section{Detection of CAIX}

CAIX initially was detected using dot blot analysis (Galperin et al., 2004; Magi \& Liberatori, 2005) to detect the optimum hybridization conditions, then detected by western blot analysis using western blot kit (BioBasic, Inc., Canada) according to the manufacturer instructions. Hybridization was performed using anti-rabbit anti-CAIX (raised against a synthetic peptide corresponding to amino-acids 432-448 of human CAIX; Thermo Fisher Scientific, USA) (1:100) over night at RT with gentle agitation. Unbound antibody washed by $1 \mathrm{X}$ PBS (supplemented with $0.05 \%$ Tween-20) and the membrane was then incubated with horseradish peroxidase labeled antirabbit antibody (1:2500) (Amersham Biosciences, UK) for 1 hour at RT with gentle agitation. After wash, the membrane was soaked in DAB $\left(3,33^{\prime}\right.$ Diaminobenzidine) substrate solution (BioBasic, Inc., Canada) and agitated for a few minutes till the bands became visible. The color development was stopped by rinsing the membrane by distilled water. The membrane was photographed and signals were scored according to the intensity.

\section{Immunological Assays}

NF- $\kappa \mathrm{B}$ p65 was detected by sandwich Enzyme Linked-Immuno-Sorbent Assay (ELISA) (BioSource International, Inc., USA) according to the manufacturer instructions. Activated NF-kB/p50 was assessed using a transcription factor assay (Rockland Immunochemicals, Inc., USA); a relatively new test which is a hybrid between ELISA and EMSA (electrophoretic mobility shift assay) where the wells are coated by a specific sequence of dsDNA (double-stranded Deoxyribonucleic acid) recognized by the transcription factor to be assessed, instead of an antibody as in a classical ELISA.

\section{Statistical Analysis}

Data analysis performed using Statistical Package for Social Sciences (SPSS) (SPSS Inc., USA) version 11.5. Normality tests showed abnormally distributed continuous variables (K.S < 0.05) except age thus it was described by mean and standard deviation $(\mathrm{M} \pm \mathrm{SD})$. Abnormally distributed variables were described using the median and range (minimum and maximum) for quantitative variables and by percentage for qualitative ones. Non-parametric statistics were applied to investigate relations between quantitative variables using the Spearman's rank correlation. 
Differences between groups regarding quantitative variables were done using the Mann Whitney U test if it was between two independent groups while Kruskal Wallis 2 2 used if it was between more than two groups. Regarding qualitative variables, the difference between groups was performed using the Fishers exact test (in case of $2 \times 2$ tables) or the Monte Carlo test (in case of rxc tables) due to invalid $\mathrm{X}^{2}$. Associations between qualitative variables were done using the 2 test, and Monte Carlo P was used due to small frequencies. All tests were two-sided and the significance was set at 0.05. The survival curve was constructed using Kaplan-Meier plots and
Wilcoxon-Gehan statistics (Kaplan \& Meier, 1958; Altman, 1991).

\section{Results}

Age of patients ranged from 20 to 74 years (minimum to maximum; $\mathrm{M} \pm \mathrm{SD}$ : 50.61 \pm 10.45 ); menopausal status: $58.1 \%$ postmenopausal, $41.9 \%$ premenopausal). Patients were treated and followed up clinically to study the impact of the studied parameters on patients' outcomes. During this period two $(2 / 31 ; 6.5 \%)$ patients had metastasis and $24 \quad(24 / 31 ; 77.41 \%)$ patients died [the median overall survival was 57 months (95\% CI 2-112 months)] (Table 1 and figure 1).

Table 1: Histopathological characteristics of patients with IDC

\begin{tabular}{|c|c|}
\hline Parameter & Number (\%) \\
\hline $\begin{array}{c}\text { Histological grade } \\
\text { II } \\
\text { III }\end{array}$ & $\begin{array}{l}18(58.1) \\
13(41.9)\end{array}$ \\
\hline $\begin{array}{l}\text { Tumor stage } \\
\text { Tx (Excision biopsy) } \\
\text { I } \\
\text { II }(A=5 ; B=4) \\
\text { III }(A=10 ; C=7) \\
\text { IV }\end{array}$ & $\begin{array}{c}1(3.2) \\
3(9.7) \\
9(29) \\
17(54.8) \\
1(3.2)\end{array}$ \\
\hline $\begin{array}{c}\text { ER? (gene expression) } \\
\text { Negative } \\
\text { Positive } \\
+ \\
++ \\
+++\end{array}$ & $\begin{array}{c}7(22.6) \\
24(77.4) \\
11(35.5) \\
3(9.7) \\
10(32.3)\end{array}$ \\
\hline $\begin{array}{c}\text { Er? (IHC) } \\
\text { Negative } \\
\text { Positive } \\
+ \\
++ \\
+++\end{array}$ & $\begin{array}{c}8(25.8) \\
23(74.2) \\
11(35.5) \\
9(29) \\
3(9.7)\end{array}$ \\
\hline $\begin{array}{r}\text { PR (total; IHC) } \\
\text { Negative } \\
\text { Positive } \\
+ \\
++ \\
+++\end{array}$ & $\begin{array}{c}8(25.8) \\
23(74.2) \\
12(38.7) \\
8(25.8) \\
3(9.7)\end{array}$ \\
\hline $\begin{array}{c}\text { Her-2/neu (gene expression)* }^{*} \\
\text { Negative } \\
\text { Positive } \\
+ \\
++ \\
+++\end{array}$ & $\begin{array}{c}8(25.8) \\
23(74.2) \\
14(45.2) \\
4(12.9) \\
5(16.1)\end{array}$ \\
\hline
\end{tabular}




\begin{tabular}{|c|c|}
\hline $\begin{array}{l}\text { LNM } \\
\text { Negative (0) } \\
\text { Positive } \\
\qquad \begin{array}{l}1(1-3) \\
\\
\qquad \begin{array}{l}(4-9) \\
(\geq 10)\end{array}\end{array}\end{array}$ & $\begin{array}{c}8(25.8) \\
23(74.2) \\
9(29) \\
7(22.6) \\
7(22.6)\end{array}$ \\
\hline $\begin{array}{l}\text { Tumor size (cm) } \\
\text { Minimum-Maximum } \\
\qquad< \\
2-4 \\
>4 \\
\text { Unknown (excision biopsy) }\end{array}$ & $\begin{array}{c}1-9 \\
2(6.5) \\
15(48.4) \\
13(41.9) \\
1(3.2)\end{array}$ \\
\hline $\begin{array}{c}\text { CAIX (protein expression) } \\
\text { Negative } \\
\text { Positive } \\
+ \\
++ \\
+++ \\
\end{array}$ & $\begin{array}{c}5(16.1) \\
26(83.9) \\
12(38.7) \\
11(35.5) \\
3(9.7) \\
\end{array}$ \\
\hline $\begin{array}{l}\text { Clinical outcome } \\
\text { Metastatic } \\
\quad \text { Local } \\
\quad \text { Distant (bone \& pleural } \\
\quad \text { effusion) } \\
\text { Death } \\
\text { DFS (disease free survival) } \\
\text { OS (overall survival) } \\
\end{array}$ & $\begin{array}{c}2(6.45) \\
0 \\
2(6.45) \\
\\
24(77.42) \\
29(93.5) \\
7(22.6) \\
\end{array}$ \\
\hline $\begin{array}{l}\text { Treatment } \\
\text { Chemotherapy (Chem) } \\
\text { Radiotherapy (Radio) } \\
\text { Hormonal therapy (Horm) } \\
\text { Chem + Radio+ Horm } \\
\text { Chem + Radio } \\
\text { Chem + Horm } \\
\text { Radio + Horm } \\
\text { Missed }\end{array}$ & $\begin{array}{l}8(25.8) \\
1(3.2) \\
2(6.5) \\
5(16.1) \\
9(29) \\
1(3.2) \\
1(3.2) \\
4(12.9)\end{array}$ \\
\hline
\end{tabular}

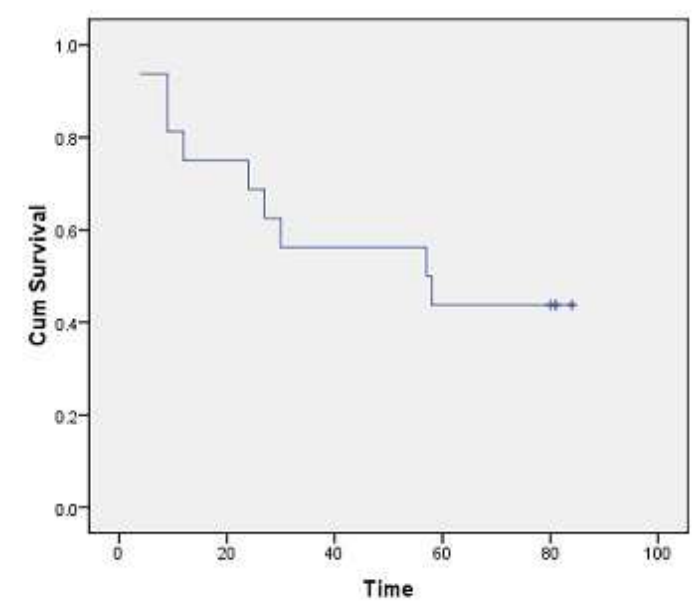

Figure 1. Overall survival of patients with invasive ductal carcinoma.

Eman El-Abd, Cecil A. Matta, Manal Sheta, Yasser El-Kerm, Mohammed Samy Afifi, Traki Benhassine, Sarah Meftahi, Shimaa Sakr and Baseem Elsherbini (2015), Advances in Cancer Research \& Treatment, 
The histopathological characteristics of patients with IDC are summarized in table1. NF-kB/P65 concentration significantly elevated $(\mathrm{p}=0.028)$ in cancerous specimens $(\mathrm{M} \pm \mathrm{SD}=$ $42.97 \pm 8.14 \mathrm{pg} / \mathrm{ml}$ ) than their normal counterparts $(\mathrm{M} \pm \mathrm{SD}=30.5 \pm 2.8 \mathrm{pg} / \mathrm{ml}$; Table 2).

Table 2: NF-? $B$ in the studied groups

\begin{tabular}{|l|l|l|}
\hline NF-? & \multicolumn{1}{|c|}{ Normal Breast tissues } & Cancerous breast tissues \\
\hline $\begin{array}{l}\text { P65 (pg/ml) } \\
\text { Median } \\
\text { Minimum-maximum }\end{array}$ & 30.5 & \\
Mean \pm SD & $30.5-35.0$ & 42.1 \\
\hline P50 (OD) & & $31.5-54.9$ \\
Median & 0.128 & $42.97 \pm 8.14^{*}$ \\
Minimum-maximum & $0.081-0.251$ & 0.091 \\
Mean \pm SD & $0.174 \pm 0.042$ & $0.081-0.307$ \\
& & $0.142 \pm 0.072$ \\
\hline
\end{tabular}

*Wilcoxon signed ranks test; $\mathrm{P}=0.028$ (significant when compared to normal breast tissues)

No significant difference $(p=0.592)$ was observed between P65 concentration and tumour stages not even after combining stages (Tx, I, II Vs III, IV) to avoid the effect of small sample size ( $p=0.950$; Table 3$)$.

Table 3: Relation between NF-? B and tumor stages (combined)

\begin{tabular}{|l|l|l|}
\hline \multirow{2}{*}{ NF-? } & \multicolumn{2}{|c|}{ Tumor stages (number of cases) } \\
\cline { 2 - 3 } & \multicolumn{1}{|c|}{ TX+I+II (13) } & \multicolumn{1}{c|}{ III+IV (18) } \\
\hline P65 (pg/ml) & 35 & 37.480 \\
Median & $28.472-152.680$ & $26.865-232.100$ \\
Range & & \\
\hline P50 (OD) & 0.131 & 0.125 \\
Median & $0.081-0.251$ & $0.081-0.193$ \\
Range & & \multicolumn{1}{|c|}{ (13) } \\
\hline
\end{tabular}

Mann-Whitney U test (for $p 65$ : $U=106 ; p=0.950$ \& for $p 50: U=96 ; p=0.632$ )

Although there was a numerical difference among various molecular subtypes of the breast cancer, the concentration of P65 did not vary significantly among them $(\mathrm{p}=$ 0.307; Table 4).

Table 4: NF-?B in IDC subtypes

\begin{tabular}{|l|l|l|l|l|}
\hline NF-[?B & \multicolumn{4}{|c|}{ IDC Subtypes } \\
\cline { 2 - 5 } & $\begin{array}{c}\text { ER? } \\
\text { positive/Her-2 } \\
\text { neu positive } \\
(\mathbf{1 8 )}\end{array}$ & $\begin{array}{c}\text { ER } \\
\text { Her-2 neu } \\
\text { negative (2) }\end{array}$ & $\begin{array}{c}\text { ER? positive/ } \\
\text { Her-2 neu } \\
\text { negative (6) }\end{array}$ & $\begin{array}{c}\text { ER? negative/ } \\
\text { Her-2 neu } \\
\text { positive (5) }\end{array}$ \\
\hline $\begin{array}{l}\text { p65 (pg/ml) } \\
\text { Median } \\
\text { Range }\end{array}$ & 33.59 & 81.01 & 38.08 & 36.41 \\
\hline
\end{tabular}




\begin{tabular}{|l|l|l|l|l|}
\hline NF-kB p50 & & 0.164 & 0.128 & 0.147 \\
(OD) & 0.106 & $0.155-0.174$ & $0.081-0.182$ & $0.114-0.192$ \\
Median & $0.081-0.251$ & & & \\
\hline
\end{tabular}

Kruskal-Wallis test ? $^{2}$ (for $\mathrm{p} 65 \mathrm{H}=3.606 ; \mathrm{p}=0.307$ \& for $\mathrm{p} 50 \mathrm{H}=3.696 ; \mathrm{p}=0.296$ )

P65 also showed no significant difference with any of tumour grade, lymph node metastasis (LNM), and the status of ER?
PR (total) or Her-2/neu or menopausal status (Table 5).

Table 5: Relation between NF-?B (p 65 \& p50) and clinic pathological parameters

\begin{tabular}{|c|c|c|c|c|c|c|}
\hline & \multicolumn{2}{|c|}{ NF-?B (p65) } & P value & \multicolumn{2}{|c|}{ NF-? $B(p 50)$} & P value \\
\hline & \multicolumn{2}{|c|}{ NF(-ing/(p/g) } & \multirow[t]{3}{*}{$P$ value } & \multicolumn{2}{|c|}{ NF-(QBए 50$)$} & \multirow[t]{3}{*}{ P value } \\
\hline & \multicolumn{2}{|c|}{ Median(ng/ml)Range } & & \multicolumn{2}{|c|}{ Median (OD)Range } & \\
\hline & Median & Range & & Median & Range & \\
\hline \multicolumn{7}{|l|}{ LNM } \\
\hline $\begin{array}{l}\text { Magapiaues(8) } \\
\text { sPatsisive (23) } \\
\text { Pre- }\end{array}$ & $\begin{array}{l}33.590 \\
39.8700 \\
36.750\end{array}$ & $\begin{array}{l}30.7-54.9 \\
26.9-232.9 \\
28.5-232.1 \\
\end{array}$ & $\begin{array}{l}U=75 \\
\mathrm{~B}=\mathbf{0} .134 \\
P=0.890\end{array}$ & $\begin{array}{l}0.117 \\
0.134 \\
0.134 \\
\end{array}$ & $\begin{array}{l}0.092-0.166 \\
0.084-0.251 \\
0.251 \\
\end{array}$ & $\begin{array}{l}U=80.5 \\
B= \\
0.11 .5\end{array}$ \\
\hline $\begin{array}{l}\text { mentepausal } \\
(1 \beta) 18) \\
\text { Pp }\{t 3) \\
\text { menopausal }\end{array}$ & $\begin{array}{l}37.96 \\
33.590\end{array}$ & $\begin{array}{l}26.9-232.1 \\
28.5-152.7\end{array}$ & $\begin{array}{l}\mathrm{U}=99 \\
P=0.489\end{array}$ & $\begin{array}{l}0.115 \\
0.155\end{array}$ & $\begin{array}{l}0.081- \\
0 . \oplus 881-0.189 \\
0.081-0.251\end{array}$ & $\begin{array}{l}P= \\
0.8286 \\
P= \\
0.042 \#\end{array}$ \\
\hline \multicolumn{7}{|l|}{ ER89:status } \\
\hline $\begin{array}{l}\text { (IHC) } \\
\text { Negative (8) } \\
\text { Positive (23) }\end{array}$ & $\begin{array}{l}35.731 \\
39.091\end{array}$ & $\begin{array}{l}29.1-48.9 \\
26.96-232.1\end{array}$ & $\begin{array}{l}U= \\
81.000 \\
P=0.619\end{array}$ & $\begin{array}{l}0.132 \\
0.128\end{array}$ & $\begin{array}{l}0.087-0.184 \\
0.081-0.251\end{array}$ & $\begin{array}{l}\mathrm{U}=86.5 \\
P= \\
0.804\end{array}$ \\
\hline $\begin{array}{l}\text { ER? status } \\
\text { (gene } \\
\text { expression) } \\
\text { Negative (7) } \\
\text { Positive (24) } \\
\end{array}$ & $\begin{array}{l}37.870 \\
35.340\end{array}$ & $\begin{array}{l}29.1-119.5 \\
26.9-232.1\end{array}$ & $\begin{array}{l}\mathrm{U}=76 \\
P=0.705\end{array}$ & $\begin{array}{l}0.155 \\
0.115\end{array}$ & $\begin{array}{l}0.114-0.192 \\
0.081-0.251\end{array}$ & $\begin{array}{l}\mathrm{U}=44 \\
P= \\
0.940\end{array}$ \\
\hline $\begin{array}{l}\text { PR status (IHC) } \\
\text { Negative (8) } \\
\text { Positive (23) }\end{array}$ & $\begin{array}{l}37.736 \\
37.091\end{array}$ & $\begin{array}{l}30.7-48.9 \\
26.9-232.1\end{array}$ & $\begin{array}{l}U= \\
91.000 \\
P=0.964\end{array}$ & $\begin{array}{l}0.132 \\
0.128\end{array}$ & $\begin{array}{l}0.087-0.192 \\
0.081-0.251\end{array}$ & $\begin{array}{l}\mathrm{U}=84.5 \\
P= \\
0.735\end{array}$ \\
\hline $\begin{array}{l}\text { Her-2/neu } \\
\text { status (gene } \\
\text { expression) } \\
\text { Negative (8) } \\
\text { Positive (23) }\end{array}$ & $\begin{array}{l}39.113 \\
33.590\end{array}$ & $\begin{array}{l}32.4-119.5 \\
26.9-232.1\end{array}$ & $\begin{array}{l}U= \\
64.000 \\
P=0.204\end{array}$ & $\begin{array}{l}0.135 \\
0.121\end{array}$ & $\begin{array}{l}0.081-0.182 \\
0.081-0.251\end{array}$ & $\begin{array}{l}U=83 \\
P= \\
0.684\end{array}$ \\
\hline
\end{tabular}

U: Mann-Whitney test, p value significant at 0.005 (2-sided).

IHC: Immunohistochemistry; LNM: Lymph Node Metastasis.

No correlation was observed with any of age, the number of the involved lymph node (LN), tumour size, the status of ER?,
PR (total) or Her-2/neu, P50 binding activity, or CAIX expression (Table 6). 
Table 6: Correlation between hypoxic markers and prognostic factors

\begin{tabular}{|c|c|c|c|}
\hline Prognostic factors & $\begin{array}{l}\text { p65 (in tumor } \\
\text { tissues) }\end{array}$ & $\begin{array}{c}\text { p50 (in tumor } \\
\text { tissues) }\end{array}$ & $\begin{array}{c}\text { CAIX (in tumor } \\
\text { tissues) }\end{array}$ \\
\hline $\begin{array}{l}\text { Age } \\
\mathbf{r} \\
\mathbf{p} \\
\end{array}$ & $\begin{array}{c}-0.192 \\
0.302 \\
\end{array}$ & $\begin{array}{c}-0.149 \\
0.424 \\
\end{array}$ & $\begin{array}{l}0.294 \\
0.109\end{array}$ \\
\hline $\begin{array}{l}\text { The number of the } \\
\text { involved LNs } \\
\text { r } \\
\text { p }\end{array}$ & $\begin{array}{c}-0.068 \\
0.717 \\
\end{array}$ & $\begin{array}{l}0.069 \\
0.711 \\
\end{array}$ & $\begin{array}{c}-0.417^{*} \\
0.020 \\
\end{array}$ \\
\hline $\begin{array}{l}\text { Tumor size } \\
\text { r } \\
\text { p }\end{array}$ & $\begin{array}{c}-0.070 \\
0.712 \\
\end{array}$ & $\begin{array}{l}0.049 \\
0.795 \\
\end{array}$ & $\begin{array}{c}-0.270 \\
0.149 \\
\end{array}$ \\
\hline $\begin{array}{l}\text { ER? status (IHC) } \\
\mathbf{r} \\
\mathbf{p}\end{array}$ & $\begin{array}{l}0.134 \\
0.471\end{array}$ & $\begin{array}{l}-0.136 \\
0.467\end{array}$ & $\begin{array}{l}0.149 \\
0.425\end{array}$ \\
\hline $\begin{array}{l}\text { ER? ? status (gene } \\
\text { expression) } \\
\mathbf{r} \\
\mathbf{p} \\
\end{array}$ & $\begin{array}{c}-0.021 \\
0.910 \\
\end{array}$ & $\begin{array}{c}-0.534^{* *} \\
0.002 \\
\end{array}$ & $\begin{array}{l}0.166 \\
0.373 \\
\end{array}$ \\
\hline $\begin{array}{l}\text { PR (IHC) } \\
\text { p } \\
\text { p }\end{array}$ & $\begin{array}{c}-0.170 \\
0.360\end{array}$ & $\begin{array}{l}0.036 \\
0.848\end{array}$ & $\begin{array}{c}-0.073 \\
0.697\end{array}$ \\
\hline $\begin{array}{l}\text { Her-2/neu status (gene } \\
\text { expression) } \\
\text { r } \\
\text { p }\end{array}$ & $\begin{array}{c}-0.192 \\
0.301 \\
\end{array}$ & $\begin{array}{r}-0.321 \\
0.078 \\
\end{array}$ & $\begin{array}{c}-0.032 \\
0.865 \\
\end{array}$ \\
\hline $\begin{array}{l}\text { P65 (in tumor tissues) } \\
\text { r } \\
\text { p }\end{array}$ & & $\begin{array}{l}0.158 \\
0.397\end{array}$ & $\begin{array}{l}0.295 \\
0.107\end{array}$ \\
\hline $\begin{array}{l}\text { P50 (in tumor tissues) } \\
\text { r } \\
\text { p }\end{array}$ & $\begin{array}{l}0.158 \\
0.397\end{array}$ & & $\begin{array}{c}-0.065 \\
0.728\end{array}$ \\
\hline $\begin{array}{l}\text { CAIX (in tumor tissues) } \\
\mathbf{r} \\
\mathbf{p}\end{array}$ & $\begin{array}{l}0.295 \\
0.107 \\
\end{array}$ & $\begin{array}{c}-0.065 \\
0.728 \\
\end{array}$ & \\
\hline $\begin{array}{l}\text { P65 (in normal tissues) } \\
\text { r } \\
\text { p }\end{array}$ & $\begin{array}{c}-0.143 \\
0.787 \\
\end{array}$ & $\begin{array}{r}-0.029 \\
0.967 \\
\end{array}$ & $\begin{array}{c}-0.131 \\
0.805 \\
\end{array}$ \\
\hline $\begin{array}{l}\text { P50 (in normal tissues) } \\
\text { r } \\
\text { p }\end{array}$ & $\begin{array}{c}-0.008 \\
0.983\end{array}$ & $\begin{array}{c}-0.126 \\
0.748\end{array}$ & $\begin{array}{l}0.550 \\
0.125\end{array}$ \\
\hline
\end{tabular}

Spearman's rho: *correlation is significant at the 0.05 level (2-tailed) \& ${ }^{* *}$ correlation is significant at the 0.01 level (2-tailed)

NF-kB/p50 binding activity did not significantly differ between cancerous specimens and their normal counterparts ( $p=0.748$; Table 2). P50 binding activity significantly elevated with higher tumour grades ( $p=0.042$; Table 5 ) but did not show any significant difference with tumour stage $(p=0.632$; Table 3$)$. A significant inverse correlation was observed between p50 and ER status (as detected by RT-PCR) ( $\mathrm{r}=-0.534, \mathrm{p}=0.002$; Table 6). P50 did not significantly vary 
among the IDC subtypes ( $p=0.296$; Table 4). It also differed in proportion of both ER/CAIX positive tumours than ER/CAIX negative tumours $(p=0.059)$ and CAIX positive than CAIX negative tumours ( $p=$ 0.068).

CAIX expression was detected in $84 \%$ of tumour specimens (Table 1 \& Figure 2) whereas; no expression was detected in normal breast tissue specimens. CAIX expression showed a significant inverse correlation with the number of the involved lymph node $(r=-0.417, \mathrm{p}=0.020$; Table 6). CAIX expression differed in proportion with tumour size $(p=0.068)$. No significant difference was observed with any other prognostic factors (Table 5). No correlation was detected between CAIX, p65, or p50 (Table 6).

a.

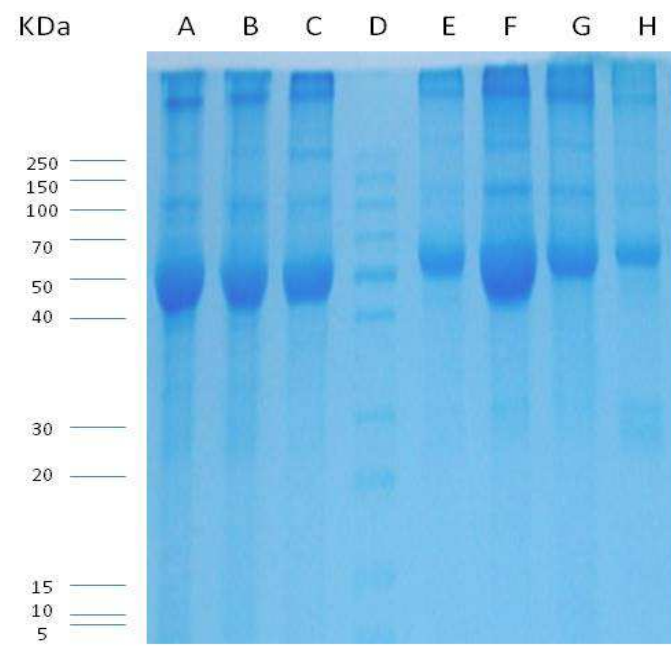

b.

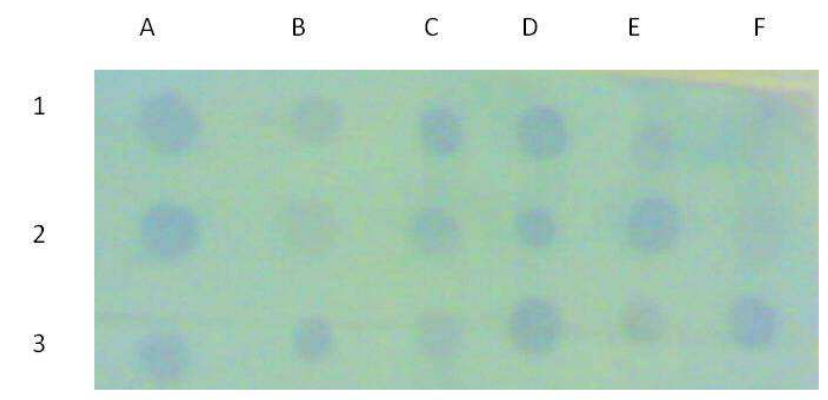

Figure 2. SDS-PAGE for representative samples of breast tissues' proteins (a) and dot blot analysis (b).

a. A-C: normal breast specimens; D: PageRuler Unstained Broad Range Protein Ladder (molecular weight of the bands are indicated on the right); E-H: tumor breast specimens.

b. A1-A3: positive control (HeLa cells); B1, B2, C3, E1, E3, F2: weak positive; B3, C2, D2: moderate positive; C1, D1, D3, E2, F3: strong positive; F1: negative (normal breast specimen).

Nottingham prognostic index (NPI) revealed that the majority of patients are of

poor NIP (Table 7) which accounts for low overall survival rate. Patients received 
proper adjuvant therapy after surgery which would account for high disease free survival (DFS) (Table 1). The only one case with good NPI was a triple negative breast cancer case. No statistical difference was observed in the hypoxic markers and the NPI groups (Table 7).

Table 7: Nottingham prognostic index (NPI), patients' outcomes, and the hypoxic markers

\begin{tabular}{|c|c|c|c|c|c|c|c|}
\hline \multirow{2}{*}{$\begin{array}{l}\text { NPI groupings } \\
\text { (score) }\end{array}$} & \multicolumn{3}{|c|}{ Outcomes } & \multirow{2}{*}{$\begin{array}{l}\mathrm{N}(\%) \text { of } \\
\text { OS }\end{array}$} & \multicolumn{3}{|c|}{ Hypoxic markers } \\
\hline & $\begin{array}{l}\text { Death } \\
\text { N (\%) }\end{array}$ & $\begin{array}{c}\text { Metastasi } \\
\text { s } \\
\text { N (\%) }\end{array}$ & $\begin{array}{l}\text { Total } \\
\text { N (\%) }\end{array}$ & & $\begin{array}{c}\mathrm{P65} \\
(\mathrm{pg} / \mathrm{ml}) \\
\mathrm{M} \pm \mathrm{SE}\end{array}$ & $\begin{array}{c}\text { P50 (OD) } \\
\text { M } \pm S E\end{array}$ & $\begin{array}{l}\text { CAIX } \\
(\mathrm{N}, \%)\end{array}$ \\
\hline Excellent $(\leq 2.4)$ & $0(0)$ & $0(0)$ & $0(0)$ & $0(0)$ & - & - & - \\
\hline Good (2.4-3.4) & $1(100)$ & $0(0)$ & $1(3.2)$ & $0(0)$ & $52.9 \pm 16.4$ & $\begin{array}{l}0.124 \pm 0.0 \\
1\end{array}$ & $\begin{array}{l}\text { Negative } 2 \\
(16.7)\end{array}$ \\
\hline $\begin{array}{l}\text { Moderate (3.4- } \\
5.4)\end{array}$ & $7(63.6)$ & $1(9.1)$ & $11(35.5)$ & $4(36.4)$ & & & $\begin{array}{ll}+ & 3 \\
(25) & \\
++ & 4 \\
(33.3) & \\
+++ & 3 \\
(25) & \\
\end{array}$ \\
\hline Poor $(>5.42)$ & $13(86.7)$ & $1(6.7)$ & $15(48.4)$ & $2(13.3)$ & $\begin{array}{l}46.80 \pm \\
7.44\end{array}$ & $\begin{array}{l}0.143 \pm 0.0 \\
5\end{array}$ & $\begin{array}{l}\text { Negative } \\
(15.8)\end{array}$ \\
\hline Very poor $(\geq 6.5)$ & $3(75)$ & $0(0)$ & $4(12.9)$ & $1(25)$ & & & $\begin{array}{ll}+ & 9 \\
(47.4) & \\
++ & 7 \\
(36.8) & \\
+++ & 0 \\
(0) & \end{array}$ \\
\hline
\end{tabular}

M: mean; N: number; OD: optical density; OS: overall survival; SE: standard error

\section{Discussion}

This is the first study to investigate the relation between p65, p50, and CAIX in IDC. The current study and others (Sovak et al., 1997; Montagut et al., 2006; Hou et al., 2003; Jana et al., 2012; Shapochka et al., 2012) showed activated p65 in human breast tissue specimens. Induction of NF? B activity and signal transduction was reported in breast cancer as a mediator of hypoxic-cellular response (Nakshatri et al., 1997; Karin et al., 2002; Biswas et al., 2004; Bendinelli et al., 2009; Madhusoodhanan et al., 2009; Tafani et al., 2011). P65 forms a predominantly active p65/p50 heterodimer in epithelial cells that is capable of switching on the cellular survival programs, and thus increases tumour proliferation and progression. It also transactivates genes involved in inflammatory response, cell cycle, and invasion; and thus enhances tumour growth and its capacity to proliferate and metastasize (Higgins et al., 1993; Tozawa et al., 1995; Sharma \& Narayanan, 1996; Shishodia \& Aggarwal, 2004; Ahn \& Aggarwal, 2005; DiDonato et al., 2012).

Controversial results were reported regarding the association between p65 and prognostic factors in breast cancer. No statistical difference was observed between p65 and tumour grade, LNM, hormonal status, Her-2/neu status, in the present and previous studies (Sovak et al., 1997; Dejardin et al., 1999; Cogswell et al., 2000; Hou et al., 2003; Montagut et al., 2006; Jana et al., 2012; Shapochka et al., 2012). Although Pikarskya and Ben-Neriah 
(2006) suggested that the activation of NF? $\mathrm{B}$ in breast cancer might be stage dependant, our results (in Egyptian population) and Hou et al., (2003) (in Taiwanese population) showed no difference between p65 and different breast tumour stages despite the stage distribution, the sample size, and detection method. In Hou's study (2003) the majority of breast cancer patients were of stage II $(68 / 82 ; 83 \%)$ while in the current study the majority of cases were of stage III $(17 / 31 ; 54.8 \%)$. However, in an Indian study (Jana et al., 2012) that included 57 breast cancer patients, p65 showed significant association with advanced stage, larger tumour size $(\geq 5 \mathrm{~cm})$, higher grade, negative ER, negative PR, positive Her-2 status, and high NPI (Nottingham prognostic index). NF-? B activation occurs via at least two different pathways depending on the stimulus (Ahn \& Aggarwal, 2005; Johnson \& Perkins, 2012). NF-?B also acts as a tumour promoter or suppressor depending on the p53 status (Johnson \& Perkins, 2012). Whether this discrepancy would reflect racial variations due to various exposures and/or other concomitant diseases or p53 status needs further investigations.

Also absence of significant difference between p65 and LNM was reported apart from the LNM status and sample size. In Hou (2003) and Montagut (2006) studies, the majority of cases were negative for LNM $[(52 / 82 ; 63.4 \%)$, and $(28 / 51 ; 55 \%)$; respectively] while the majority of our cases were positive (23/31; 74.2\%). Although no relation was observed between CAIX and LNM status in the current study and others (Chia et al., 2001; Tan et al., 2009), an inverse significant correlation was observed between CAIX and the number of the involved lymph nodes reflecting a possible protective role of CAIX in subset of patients with IDC breast cancer. In contrast, in a study containing 253 breast cancer tissue samples, this relation was absent (Span et al., 2003). This might be due to the detection method (protein versus gene expression), and/or sample size (31 versus 253). Larger cohort study is mandatory to further investigate a possible dual role of CAIX in breast cancer.

Although no relation was observed between elevated level of nuclear p65 or CAIX and tumor grade, a significant relation was observed with p50. The expression of the p50 rate was higher in tumors of higher grade (i.e. with high proliferative potential). This result is consistent with that previously reported by Hou et al., (2003). Absence of relation with p65 and presence of it with p50 would reflect the dominance of p50 heterodimers $(\mathrm{p} 50) 2$ in the examined specimens. (P50)2 is known to be able to work as a transcriptional regulator on genes on both repressed and active chromatins and act as transcriptional repressor or activator depending on the cellular protein pools and stimuli (Koong et al., 1994).

The current study further support the translocation of p65 regardless the ER, PR, and Her-2/neu status and hence the IDC subtypes. These results are in accordance with that reported by Cogswell et al., (2000); and Montagut et al., (2006). In contrast, several reports (Nakshatri et al., 1997; Biswas et al., 2005; Zhou et al., 2005a \& b; Singh et al., 2007) have demonstrated that higher levels of NF-kB are associated with ER negative status than in ER-positive in cells and tumours and most predominantly in ER- negative/Her2/neu- positive breast tumours. However, the P65 nuclear concentration in the present study is numerically higher in ERpositive than ER- negative tumours (53.5 versus $36.7 \mathrm{pg} / \mathrm{ml}$; respectively). Absence of the relation between p65 level and ERstatus in the current study might be due to the fact that most of the breast cancer tumours were ER- positive $(23 / 31 ; 74 \%)$ and the ER- negative/Her-2/neu- positive were 5/31 (16\%; with $3 / 31$ with Her$2 /$ neu overexpression). In addition, controversial results would be explained by the fact that the mutually antagonistic cross-talk between the NF-?B and ER signalling pathways occurs via various mechanisms which might be stimulus or cell type specific.

The malignant progression of some breast cancers, however, is coincident with a shift 
from estrogen dependence to estrogen independence and, notably, this shift also coincides with an increase in both NF-? B activities (Nakshatri et al., 1997; Pratt et al., 2003) and the expression of some NF-? ? target genes (Freund et al., 2004). It has been also shown that Her-2/neu expression leads to down regulation of ER and activation of NF-?B (Ahmed et al., 2006). Thus higher levels of p65 in ER- positive tumours than normal breast specimens (53.5 versus $30.5 \mathrm{pg} / \mathrm{ml}$ ) might indicate a pre-transition state towards loss of ER and acquisition of Her-2/neu overexpression. However, further studies are mandatory to prove this hypothesis.

Interestingly, although no significant difference was observed between the p50 binding activity in tumor and their normal counterparts, a significant inverse relation was observed between ER and p50 subunit in tumor specimens in the present and previous studies (Nakshatri et al., 1997; Biswas et al., 2004). The activation rate of the p50 was numerically higher in patients with ER $\alpha$-negative status, whereas patients with ER $\alpha$ positive/Her-2 positive status maintained a low activation rate. Similar results were previously reported by Zhou et al., (2005a \& b) and then explained by the presence of Her-2, significantly correlated with the degree of binding of p50 and p65.

The expression rate of p50 in ER $\alpha$-negative tumors (regardless the status of Her- 2 in seven of our samples) suggest the activation of p50 which is close to the data obtained by Biswas et al., (2004) and may foreshadow increased therapeutic resistance for these patients, as suggested by Pratt et al., (2003).

Three cases expressed higher levels of p65 ( $>100 \mathrm{pg} / \mathrm{ml}$ ). Two cases out of the three cases $(2 / 3 ; 66.7 \%)$ showed edema, peou d' orange, presence of prominent stromal inflammatory infiltrate, and presence of lymphovascular invasion which represent IBC (Inflammatory Breast Cancer) clinical signs. Many investigators (Van Laere et al., 2005 \& 2007; Lerebours et al., 2008; Ahmed, 2010) reported that the NF-? B signature of IBC offers an explanation for the inflammatory symptoms because NF? $\mathrm{B}$ is an activator of several inflammatory genes including chemotactic genes, growth factors, angiogenic factors, and matrix metalloproteinase, which are responsible for metastasis, cell proliferation, and angiogenesis, processes known to belong to the biology of IBC.

The limited number of IBC cases in the current study could also explain the absence of significance between [NF-? B] and other histopathological parameters. One of the two cases with IBC in our study was ER+, Her-2+, [NF- ? B = 152 pg/ml] still alive. The other case was ER-, Her-2-, [NF? $\mathrm{B}=119 \mathrm{pg} / \mathrm{ml}$ ] and had history of breast sarcoma which recurred as IDC followed by death within one year of the elective surgery. This might add further possible differential role in metastatic IBC than non metastatic IBC which might be confirmed in the future studies.

\section{Conclusion}

Although no relation was observed between p65, p50, and CAIX, binding activity of p50 and CAIX concentration might be used as prognostic markers in IDC. Patients with elevated level of CAIX would benefit from CAIX inhibitors (as adjuvant therapy) and imaging probes (in diagnosis and follow up). Similarly, patients with elevated level of p50 would benefit from natural (such as piperlongumine, alpha tomatine...etc) and synthetic p50 antagonists.

\section{References}

1. Ahmed, A. (2010) "Prognostic and therapeutic role of nuclear factor-kappa B (NF-kappaB) in breast cancer," J Ayub Med Coll Abbottabad, 22 218-21.

2. Ahmed, K.M., Cao, N. and Li, J. J. (2006) "HER-2 and NF-kappaB as the targets for therapy-resistant breast cancer," Anticancer Res, 26 4235-43.

3. Ahn, K. S. and Aggarwal, B. B. (2005) "Transcription Factor NF-?B A Sensor for 
Smoke and Stress Signals," Ann NY Acad Sci, 1056 218-33.

4. Altman, D.G. Practical statistics for medical research. London: Chapman and Hall; 1991. Pp. 403-5.

5. Bartosova, M., Parkkila, S., Pohlodek, K., Karttunen, T.J., Galbavy, S., Mucha, V., et al. (2002) "Expression of carbonic anhydrase IX in breast is associated with malignant tissues and is related to overexpression of c-erbB2," J Pathol, 197 314-21.

6. Basak, S. and Hoffmann, A. (2008) "Crosstalk via the NF-кB Signaling System," Cytokine Growth Factor Rev, 19 187-97.

7. Bendinelli, P., Matteucci, E., Maroni, P. and Desiderio, M. A. (2009) "NF-kappaB activation, dependent on acetylation/deacetylation, contributes to HIF-1 activity and migration of bone metastatic breast carcinoma cells," Mol Cancer Res, 7 1328-1341.

8. Biswas, D.K., Cruz, A.P., Gansberger, E. and Pardee, A.B. (2000) "Epidermal growth factor-induced nuclear factor kappa B activation: A major pathway of cell-cycle progression in estrogen-receptor negative breast cancer cells," Proc Natl Acad Sci U S A, 97(15) 8542-7.

9. Biswas, D.K., Dai, S.C., Cruz, A., Weiser, B., Graner, E. and Pardee, A. B. (2001) "The nuclear factor kappa B (NF-kappa B): a potential therapeutic target for estrogen receptor negative breast cancers," Proc Natl Acad Sci USA, 98 10386-91.

10.Biswas, D.K., Shi, Q., Baily, S., Strickland, I., Ghosh, S., Pardee, A.B., et al. (2004) "NFkappa $B$ activation in human breast cancer specimens and its role in cell proliferation and apoptosis," Proc Natl Acad Sci USA, 101 10137-42.

11.Biswas, D.K., Singh, S., Shi, Q., Pardee, A. B. and Iglehart, J. D. (2005) "Crossroads of estrogen receptor and NF-kappaB signaling," Sci STKE, 2005 pe27.

12.Bloom, H.J.G., and Richardson, W.W. (1957) "Histological grading and prognosis in breast cancer. A study of 1409 cases of which 359 have been followed for 15 years," Br J Cancer, 11 359-77.

13.Chia, S.K., Wykoff, C.C., Watson, P.H., Han, C., Leek, R.D, Pastorek J, et al. (2001) "Prognostic significance of a novel hypoxiaregulated marker, carbonic anhydrase IX, in invasive breast carcinoma," Clin Oncol, $193660-8$.

14.Cogswell, P.C., Guttridge, D.C., Funkhouser, W. K. and Baldwin, Jr. A. S. (2000) "Selective activation of NF-?B subunits in human breast cancer: potential roles for NF-?B2/p52 and for Bcl-3," Oncogene, 19 1123-31.

15.Culver, C., Sundqvist, A., Mudie, S., Melvin, A., Xirodimas, D. and Rocha, S. (2010) "Mechanism of hypoxiainduced NF-kappaB," Mol Cell Biol, 30(20) 4901-21.

16.Dejardin, E., Deregowski, V., Chapelier, M., Jacobs, N., Gielen, J. and Merville, M. P. (1999) "Regulation of NF-?B activity by I $\mathrm{O} B-$-related proteins in adenocarcinoma cells," Oncogene, 18 1835-41.

17.Déry, M.A., Michaud, M. D. and Richard, D. E. (2005) "Hypoxia-inducible factor 1: regulation by hypoxic and non-hypoxic activators," Int J Biochem Cell Biol, 37 53540.

18.DiDonato, J.A., Mercurio, F. and Karin, M. (2012) "NF- $\mathrm{KB}$ and the link between inflammation and cancer," Immunol Rev, $246379-400$.

19.El-Abd, E., Hassan, A., Faied, W., Zaki, S., Sobhi, A. and El-Swedy, S. (2012) "Clinical relevance of HIF-10, Cox-2, leptin, and prolactin as hypoxic markers in breast cancer," Austral-Asian J Cancer, 11 169-174.

20.El-Abd, E., Matta, C.A., Sheta, M., ElKerm, Y. and Sakr, S. (2014) "Histopathological characteristics of breast cancer and evaluation of ER alpha and Her2neu using immunohistochemical and RTPCR techniques," Alex J Med, http://dx.doi.org/10.1016/j.ajme.2014.03. 001. 
21.Favaro, E., Lord, S., Harris, A. L. and Buffa, F. M. (2011) "Gene expression and hypoxia in breast cancer," Genome Med, 3 55.

22.Freshney, R. I. (2010) Culture of animal cells: A manual of basic technique and specialized applications ( $6^{\text {th }}$ ed.), John Wiley \& Sons, Inc, Canada.

23.Freund, A., Jolivel, V., Durand, S., Kersual, N., Chalbos, D., Chavey, .C, et al. (2004) "Mechanisms underlying differential expression of interleukin-8 in breast cancer cells," Oncogene, 23 6105-14.

24.Galperin, M.M., Traicoff, J.L., Ramesh, A., Freebern, W.J., Haggerty, C.M., Hartmann, D.P., et al. (2004) "Multimembrane dotblotting: a cost-effective tool for proteome analysis," Biotechniques, 36 1046- 51.

25.Greene, F.L., Page, D.L., Fleming, I.D., Fritz, A., Balch, C.M., Haller, D. G., et al., editors. (2002) AJCC cancer staging manual. 6th ed. New York: Springer.

26.Gupta, S.C., Sundaram, C., Reuter, S. and Aggarwal, B.B. (2010) "Inhibiting NF$\kappa \mathrm{B}$ activation by small molecules as a therapeutic strategy," Biochim Biophys Acta, 1799 (10-12) 775-87.

27.Haybittle, J.l., Blamey, R.W., Elstonc, W. Johnson, J., Doyle, P.J., Campbell, F.C. et al. (1982) "A prognostic index in primary breast cancer," Br J Cancer, 45 361-6.

28.Higgins, K.A., Perez, J.R., Coleman, T.A., Dorshkind, K., McComas, W.A., Sarmiento, U.M., et al. (1993) "Antisense inhibition of the p65 subunit of NF-kappa B blocks tumorigenicity and causes tumor regression," Proc Natl Acad Sci USA, 90 9901-5.

29.Hou, M.F., Lin, S.B., Yuan, S.S., Tsai, S.M., $\mathrm{Wu}$, S.H., Ou-Yang, F., et al. (2003) "The clinical significance between activation of nuclear factor kappa B transcription factor and overexpression of HER-2/neu oncoprotein in Taiwanese patients with breast cancer," Clin Chim Acta, 334 137-44.
30.Hussain, S.A., Ganesan, R., Reynolds, G., Gross, L., Stevens, A., Pastorek, J., et al. (2007) "Hypoxia-regulated carbonic anhydrase IX expression is associated with poor survival in patients with invasive breast cancer," Br J Cancer, 96 104-9.

31.Jana, D., Das, S., Sarkar, D.K., Mandal, S., Maji, A. and Mukhopadhyay, M. (2012) "Role of nuclear factor- $\kappa \mathrm{B}$ in female breast cancer: A study in Indian patients," Asian Pacific J Cancer Prev, 13 5511-15.

32.Johnson, R. F. and Perkins, N. D. (2012) "Nuclear factor-?B, p53, and mitochondria: regulation of cellular metabolism and the Warburg effect," Trends Biochem Sci, 37 317-24.

33.Kaluz, S., Kaluzová, M., Chrastina, A., Olive, P.L., Pastoreková, S., Pastorek, J., et al. (2002) "Lowered oxygen tension induces expression of the hypoxia marker $\mathrm{MN} /$ carbonic anhydrase IX in the absence of hypoxia-inducible factor 1 alpha stabilization: a role for phosphatidylinositol 3'-kinase," Cancer Res, 62(15) 4469-77.

34.Kaplan, E. L., and Meier, P. (1958). "Nonparametric estimation from incomplete observations," J Amer Statist Assn, 53 (282) 457-481.

35.Karin, M., Cao, Y., Greten, F. R. and Li, Z. W. (2002) "NF-kappaB in cancer: from innocent bystander to major culprit," Nat Rev Cancer, 2 301-10.

36.Kenneth, N. S. and Rocha, S. (2008) "Regulation of gene expression by hypoxia," Biochem J, 414 (1) 19-29.

37.Knowles, H. J. and Harris, A. L. (2001) "Hypoxia and oxidative stress in breast cancer: Hypoxia and tumourigenesis," Breast Cancer Res, 3 318-22.

38.Koong, A.C., Chen, E. Y. and Garccia, A. J. (1994) "Hypoxia causes the activation of nuclear factor $\mathrm{kB}$ through the phosphorylation of $\mathrm{IkB} \alpha$ on tyrosine residues," Cancer Res, 54 1425-30. 
39.Lerebours, F., Vacher, S., Andrieu, C., Espie, M., Marty, M., Lidereau, R., et al. (2008) "NF-kappa B genes have a major role in inflammatory breast cancer," $B M C$ Cancer, 841.

40.Madhusoodhanan, R., Natarajan, M., Veeraraghavan, J., Herman, T. S. and Aravindan, N. (2009) "NFkappaB activity and transcriptional responses in human breast adenocarcinoma cells after single and fractionated irradiation," Cancer Biol Ther, 8 765-773.

41.Magi, B. and Liberatori, S. (2005) "Immunoblotting techniques," Methods Mol Biol, 259 227- 54.

42.May, M.J. and Ghosh, S. (1997) "Rel/NFkappa B and I kappa B proteins: an overview," Semin. Cancer Biol., 8:63-73.

43. Montagut, C., Tusquets, I., Ferrer, B., Corominas, J.M., Bellosillo, B. and Campas, C. (2006) "Activation of nuclear factorkappa B is linked to resistance to neoadjuvant chemotherapy in breast cancer patients," Endocr Relat Cancer, 13: 607-16.

44.Nakshatri, H., Bhat-Nakshatri, P., Martin, D.A., Goulet, R. J. Jr. and Sledge, G. W. Jr. (1997) "Constitutive activation of NF-?B during progression of breast cancer to hormone-independent growth," Mol Cell Biol, 17 3629-39.

45. Nogradi, A. (1998) "The role of carbonic anhydrases in tumors," Am J Pathol, 153 279-89.

46.Parks, S.K., Chiche, J. and Pouyssegur, J. (2011) "pH control mechanisms of tumor survival and growth," J Cell Physiol, 226 299-308.

47.Pereira, S. G. and Oakley, F. (2008) "Nuclear factor-?B1: Regulation and function," Int J Biochem Cell Biol, 40 142530 .

48.Pikarsky, E. and Ben-Neriah, Y. (2006) "NF-kappaB inhibition: a double-edged sword in cancer?," Eur J Cancer, 42 779-84.
49.Pratt, M.A., Bishop, T.E., White, D., Yasvinski, G., Ménard, M., Niu, M.Y., et al. (2003) "Estrogen withdrawal-induced NF? $\mathrm{B}$ activity and $\mathrm{Bcl}-3$ expression in breast cancer cells: roles in growth and hormone independence," Mol Cell Biol, 23 6887-900.

50.Robey, I.F., Lien, A.D., Welsh, S.J., Baggett, B. K. and Gillies, R. J. (2005) "Hypoxia-inducible factor-1alpha and the glycolytic phenotype in tumors," Neoplasia, 7 324-30.

51.Sambrook, J., Fritsch, E. F. and Maniatis, T. (1989) Molecular cloning: A laboratory manual (2 $2^{\text {nd }}$ ed.), Cold Spring Harbor University Press, NY.

52.Shapochka, D.O., Zaletok, S. P. and Gnidyuk, M. I. (2012) "Relationship between NF-?B, ER, PR, HER2/NEU, KI67, P53 expression in human breast cancer," Exp Oncol, 34 358-63.

53.Sharma, H. W. and Narayanan, R. (1996) "The NF-kappaB transcription factor in oncogenesis," Anticancer Res, 16 589-96.

54.Shishodia, S. and Aggarwal, B. B. (2004) "Nuclear factor-kappaB activation mediates cellular transformation, proliferation, invasion angiogenesis and metastasis of cancer," Cancer Treat Res, 119 139-73.

55.Singh, S., Shi, Q., Bailey, S.T., Palczewski, M.J., Pardee, A.B., Iglehart, J.D., et al. (2007) "Nuclear factor-?B activation: a molecular therapeutic target for estrogen receptornegative and epidermal growth factor receptor family receptor-positive human breast cancer," Mol Cancer Ther, 6 1973-82.

56.Sovak, M.A., Bellas, R.E., Kim, D.W., Zanieski, G.J., Rogers, A.E., Traish, A.M., et al. (1997) "Aberrant nuclear factorkB/rel expression and the pathogenesis of breast cancer" J Clin Invest, 100 2952-60.

57.Span, P.N., Bussink, J., Manders, P., Beex, L. V. A. M, and Sweep C. G. J. (2003) "Carbonic anhydrase-9 expression levels and prognosis in human breast cancer: association with treatment outcome," $\mathrm{Br} J$ Cancer, 89 271-6. 
58.Tafani, M., Schito, L., Pellegrini, L., Villanova, L., Marfe, G., Anwar, T., et al. (2011) "Hypoxia-increased RAGE and P2X7R expression regulates tumor cell invasion through phosphorylation of Erk1/2 and Akt and nuclear translocation of NF-\{kappa\}B," Carcinogenesis, 32 116775 .

59.Tafreshi, N.K., Bui, M.M., Bishop, K., Lloyd, M.C., Enkemann, S.A., Lopez, A.S., et al. (2012) "Noninvasive detection of breast cancer lymph node metastasis using carbonic anhydrases IX and XII targeted imaging probes," Clin Cancer Res, 18 20719.

60.Tan, E.Y., Yan, M., Campo, L., Han, C., Takano, E., Turley, H., et al. (2009) “The key hypoxia regulated gene CAIX is upregulated in basal-like breast tumors and is associated with resistance to chemotherapy," Br J Cancer, 100 405-11.

61.Taylor, C.T., and Cummins, E.P. (2009) "The role of NF-kappaB in hypoxia-induced gene expression," Ann N Y Acad Sci 1177 178-84.

62.Thiry, A., Dogné, J.M., Masereel, B. and Suporan, C. T. (2006) "Targeting tumorassociated carbonic anhydrase IX in cancer therapy," Trends Pharmacol Sci, 27 566-73.

63.Tozawa, K., Sakurada, S., Kohri, K. and Okamoto, T. (1995) "Effects of anti-nuclear factor kappa B reagents in blocking adhesion of human cancer cells to vascular endothelial cells," Cancer Res, 55 4162-7.

64.Trastour, C., Benizri, E., Ettore, F., Ramaioli, A., Chamorey, E., Pouyssegur, J., et al. (2007) "HIF-1 $\alpha$ and CAIX staining in invasive breast carcinomas: prognosis and treatment outcome," Int J Cancer, 120 1451-8.

65.Van Laere, S., Van der Auwera, I., Van den Eynden, G.G., Fox, S.B., Bianchi, F., Harris, A.L., et al. (2005) "Distinct molecular signature of inflammatory breast cancer by cDNA microarray analysis," Breast Cancer Res Treat, 93 237-46.
66.Van Laere, S.J., Van der Auwera, I., Van den Eynden, G.G., van Dam, P., Van Marck, E.A., Vermeulen, P.B., et al. (2007) "NFkappaB activation in inflammatory breast cancer is associated with oestrogen receptor downregulation, secondary to EGFR and/or ErbB2 overexpression and MAPK hyperactivation," $\mathrm{Br} J$ Cancer, 97 659-69.

67.World Health Organization. (2003) Tumors of the Breast and Female Genital Organs. Oxford [Oxfordshire]: Oxford University Press.

68.Wu, J. T. and Kral, J. G. (2005) "The NF$\mathrm{kB}$ signaling system: a molecular target in breast cancer therapy," J Surg Res, 123 15869.

69.Wycoff, C.C., Beasley, N.J.P., Watson, P.H., Turner, K.J., Pastorek, J., Sibtain, A, et al. (2000) "Hypoxia-inducible expression of tumor-associated carbonic anhydrases," Cancer Res, 60 7075-83.

70.Wykoff, C.C., Beasley, N., Watson, P.H., Compo, L., Chia, S.K., English, R., et al. (2001) "Expression of the hypoxiainducible and tumor-associated carbonic anhydrases in ductal carcinoma in situ of the breast," Am J Pathol, 158 1011-9.

71.Yde, C.W., Emdal, K.B., Guerra, B. and Lykkesfeldt, A. E. (2012) NFкB signaling is important for growth of antiestrogen resistant breast cancer cells," Breast Cancer Res Treat, 135 67-78.

72.Zhou, Y., Eppenberger-Castori, S., Marx, C., Yau, C., Scott, G.K., Eppenberger, U., et al. (2005a) "Activation of nuclear factorkappaB (NFkappaB) identifies a high-risk subset of hormone-dependent breast cancers," Int J Biochem Cell Biol, 37 113044.

73.Zhou, Y., Eppenberger-Castori, S., Eppenberger, U. and Benz, C. C. (2005b) "The NF-?B pathway and endocrine resistance," Endocr Relat Cancer, 12 (Suppl 1) S37-46. 